\title{
COVID-19: An Advisory for a Radiation Oncology Department Pertinent to the Multidisciplinary Team
}

\section{Introduction}

The cases of COVID-19 are on a rise worldwide and have posed a challenge to our nation in terms of healthcare resources, logistics, infrastructural needs, and personnel. However, patients undergoing cancer treatment require multiple visits to the hospital, are immunocompromised, often have a deranged metabolic profile, and considering all these, they could be at a higher risk. ${ }^{[1]}$ Hence, it is important to keep in mind the increased risk of being infected by COVID-19 for this subgroup of patients, while planning their treatment during the pandemic. In fact, a study conducted by a tertiary center at Wuhan showed that cancer as comorbidity itself causes the mortality due to COVID-19 to be as high as $25 \%{ }^{[2]}$ At the same time, treatment of a significant proportion of cancer patients should be done on a priority basis failing which those patients are at a higher risk of cancer-related mortality. A cancer patient undergoing concurrent chemotherapy and radiotherapy (RT) is one of the most vulnerable patients.

To strike a balance between the enhanced risks among COVID-19 infections, the progression and mortality due to cancer and the constrained resources and professionals remain the biggest challenge in the current setting.

RT is an important part in the treatment of most solid tumors and most requiring radiation at some point in time; the intent could be radical/curative, adjuvant, or palliative. Further, very often, the radiation is delivered in a concurrent setting with chemotherapy.

There are guidelines considering delaying or deferring radiation therapy where the benefits are small or controversial. ${ }^{[3]}$ An effective guideline has been formulated to assess risk and stratify patients requiring $\mathrm{RT}^{\left[{ }^{[4]}\right.}$

It is necessary to make consideration for shorter fractionation protocols wherever feasible because it can reduce the exposure to the concerned personnel, such as healthcare worker as well as patients. The purpose of our article is to give an outline on how to optimize treatment strategy without losing benefits. Immunocompromised status is not the only risk factor in cancer patients. Other comorbidities such as patients more than 60 years of age, preexisting cardiac comorbidities, preexisting pulmonary disease, hypertension, and diabetes mellitus also pose a significant challenge.

Objective of the article

This article aims at giving a perspective of radiation therapy treatment and strategies aiming to give an overview to our medical oncology and surgical oncology colleagues to help them make decisions regarding treatment protocols.

The issues are manifold. Apart from the risks to the patients, other issues are shortage of staff in a radiation oncology department and possibility of nonavailability of healthcare workers in case of unfortunate contacts. As a result, they are forced to stay quarantined at home. Considering these facts, we may revisit our strategy for treating patients with radiation.

\section{Key Questions}

Do we have adequate staff in the department in case of a crisis, to complete treatment of patients already on radiotherapy?

A thorough evaluation should be made of patient enrolments, and a department should consider that even if $40 \%-50 \%$ of its staff are quarantined or unavailable, at least the treatment of existing patients in the department can go on uninterrupted.

What are the general recommendations for radiation oncology units?

All steps taken should have social distancing, wearing of appropriate personal protective equipment (PPE), and sanitization as their mandatory criteria.

\section{At outpatient department}

- Safe distance of at least $1 \mathrm{~m}$ (3 feet) between individuals should be maintained

- Recent history of travel to COVID-19-infected area or proximity with a person known to have been diagnosed with COVID-19 should have been asked for

- To check for any influenza-like symptoms in all patients.

\section{Guideline for healthcare personnel}

- To emphasize for clean, trained, and dedicated staff, staff should be encouraged to use a hand sanitizer frequently and appropriately

- Shift postings

- Staff separated by time and location

- Provision of appropriate PPE with hand gloves, N95 masks, and aprons for all staff

- Screening of staff.

\section{Criterion to suspect a COVID-19 case}

Suspicion should be in line with the guidelines by the Indian Council of Medical Research or specific guidelines provided by the state. 


\section{What should be the initial evaluation policy?}

- RT treatment should be considered based on diagnosis, prognosis, and urgency to initiate treatment

- Hypofractionation treatments have benefits in breast, prostate, and lung cancer and to be used in the appropriate scenario

- Consider short-course palliative RT treatment for symptomatic relief. Regimens may be weekly once fractionation, single fraction, or with a short course of up to 5 fractions $(\mathrm{Fx})$.

\section{Policy during planning of the patient}

Whenever treating patients, all the team members should take proper precaution and that should start from the first contact point. Technologists should use N95 mask, head gear, and gloves during planning and simulation where maintaining safe distance is not possible.

\section{Can we prioritize patients?}

The NHS in its publication has categorized patients based on priority. ${ }^{[5]}$ Similarly, the Association of Radiation Oncologists of India has their guidelines. Similar guidelines have been mentioned here for the benefit of our multidisciplinary team.

\section{Categorization}

It is important to prioritize patients for radiation enrolment based on the resource availability, need, and urgency. We propose some examples which give an overview of setting priorities for treatment enrolment into radiation.

1. A radical chemoradiation for Stage II or III cervix with a radical/curative intent where chemoradiation is the primary modality - High priority

2. A chemoradiation/radiation protocol for head-and-neck cancers (oropharynx/larynx) where the intent is curative and waiting may result in stage progression - High priority

3. RT for spinal cord compression with potential neurological recovery - High priority

4. Early glottic cancer where radiation is the primary modality of treatment - Moderate priority

5. Palliation of symptoms where radiation can be effective and burden on other services can be decreased like hemoptysis in lung cancer - Moderate priority

6. Low-risk completely resected tumor - Low priority

7. RT for low-risk prostate cancer - Low priority.

\section{Can we shorten treatment protocols?}

Hypofractionation should be considered wherever possible. The strategies for different subsites have been mentioned below.

\section{Site-wise summary of the treatment with radiation}

Based on the literature search, policies, available guidelines, and resources, we propose the following strategies for a few subsites with respect to patient selection and their radiation treatment.

\section{Strategies for gastrointestinal malignancies}

Tchelebi et al. ${ }^{[6]}$ have elaborated on best practice guidelines for RT in gastrointestinal malignancies. We summarize our recommendations based on this publication and other available resources.

Esophagus

For operable patients, concurrent chemoradiotherapy (CRT) with $41.4 \mathrm{~Gy} / 23 \mathrm{Fx}$ can be used. In appropriately selected patients, 40 Gy in 15 Fx with concurrent chemotherapy with carboplatin and paclitaxel could be used. In case a patient is inoperable, definitive CRT with $50 \mathrm{~Gy} / 25 \mathrm{Fx}$ or a moderate hypofractionated schedule may be used. Dose escalation beyond 50 Gy equivalent may be avoided in the current setting. For palliation of symptoms such as bleeding and dysphagia, a schedule of $20 \mathrm{~Gy}$ in $5 \mathrm{Fx}$ or single fraction schedule wherever appropriate appears reasonable. Protracted fractionation in this setting is avoidable.

\section{Gastric}

RT may be avoided in operable and resected cases. Short fractionation schedules may be used for palliation.

Liver

Stereotactic body radiotherapy (SBRT) is an effective treatment option for hepatocellular carcinoma (48-60 Gy/3-5 Fx) and oligometastases in liver (16-45 Gy/1-5 $\mathrm{Fx})$.

\section{Pancreas}

In the current setting, adjuvant RT for resected pancreatic cancer may be avoided. For borderline pancreatic cancers after neoadjuvant chemotherapy (NACT), if the patient continued to be borderline resectable, SBRT is an effective option.

\section{Rectal}

Based on the available evidence, short-course RT (25 Gy/5 Fx) is recommended in the neoadjuvant treatment of rectal cancer whenever feasible after induction chemotherapy.

\section{Anal canal}

For non-metastatic cases when concurrent chemotherapy is used, standard fractionation schedules are appropriate. In case of no concurrent chemotherapy, a moderately hypofractionated schedule to a dose of 50 Gy/20 Fx may be used.

\section{Breast malignancies}

A modification of radiation oncology treatment was suggested in a study by Gay et al. in the backdrop of a natural disaster. ${ }^{[7]}$ In the current setting, the literature errs toward shortening of breast RT treatment protocols.

We suggest the following in the practice of breast cancer RT based on the available literature. 
- Omission of RT in patients older than 65 years or younger with significant comorbidities with invasive ductal carcinoma $\leq 3 \mathrm{~cm}$, ER/PR-positive, Her2-negative, margin-free, grade 1-2, node-negative, planned for endocrine treatment ${ }^{[8]}$

- Boost RT may be omitted in select patients above 40 years of age or younger patients with relevant comorbidities $^{[9]}$

- Moderate hypofractionated RT could be used for breast or chest wall and nodal RT, e.g., $40 \mathrm{~Gy} / 15 \mathrm{Fx}$ over 3 weeks followed by boost as merited ${ }^{[10]}$

- 28-30 Gy radiation in once a week Fx over 5 weeks or $26 \mathrm{~Gy} / 5 \mathrm{Fx}$ daily over 1 week following the data of FAST and FAST-forward trials, respectively, might be considered for node-negative tumors. ${ }^{[11,12]}$

Head and neck

Oral cavity

Early resectable

Surgery should be offered keeping in mind all precautions. In the presence of high-risk factors such as margin positivity and perinodal extension, concurrent CRT should be considered as there is a definite overall survival benefit. ${ }^{[13]}$

Locally advanced

Considering that the waiting period in hospitals for major surgeries could be around 4-6 weeks, ${ }^{[14]}$ NACT may be considered in the current scenario for borderline resectable tumors. Taxane, cisplatin, fluorouracil (TPF) regimen for 3-4 cycles should be considered before radical surgery. NACT can be used in the resectable oral cavity tumors in this COVID situation. A study showed a diminished number of patients who required mandibulectomy or adjuvant RT in the NACT arm of resectable oral cavity tumors. ${ }^{[15]}$ Thus, NACT could be considered with weekly or 3-weekly paclitaxel and carboplatin. If patients are not fit for the above-mentioned chemotherapy, metronomic chemotherapy with erlotinib or methotrexate with celecoxib can be given and surgery can be performed after 6-8 weeks.

\section{Oropharynx}

Early stage

Both surgery and RT are options; however, a single-modality RT is the preferred option. Transoral robotic surgery has been compared with RT in a phase II randomized study including 68 patients. ${ }^{[16]}$ Progression-free survival and overall survival are similar though the study was not powered to compare those outcomes. Swallowing-related quality of life at 1 year was the primary end point, which was proven to be better in the RT arm.

Locally advanced

Concomitant CRT remains the standard of care. ${ }^{[17]}$ If the patient is fit for cisplatin, consider cisplatin-based
CRT. Otherwise, carboplatin-based CRT or cetuximab/ nimotuzumab-based CRT may be considered.

Larynx and hypopharynx

For locoregionally advanced hypopharyngeal disease suitable for organ preservation, NACT may be considered. However, if the patient is fit, concurrent CRT is the treatment of choice. ${ }^{[18]}$

Nasopharynx

NACT followed by CRT (intensity-modulated RT using cisplatin) is the preferred treatment for Stage II-IV disease. As TPF needs continuous 5-FU which can increase hospital stay, alternate schedule such as gemcitabine + cisplatin may be considered. Gemcitabine and cisplatin were not tested in NACT setting in comparison with TPF regimen. However, in palliative setting, gemcitabine and cisplatin have better outcome than cisplatin and 5-FU regimen. ${ }^{[19]}$ For Stage I disease, RT is the treatment of choice.

Other general principles for head-and-neck cancers

Although CRT is the standard treatment for most nonoral cavity, i.e., locoregionally advanced tumors, the use should be weighed in the elderly patient with significant comorbidities.

Evaluation of p16 status in oropharyngeal tumors is not mandatory as this does not influence the subsequent management.

Evaluation of laryngeal function before the use of organ conservation protocols should be carried out with discretion preferring techniques such as modified Barium swallow to other invasive methods.

Further, aerosol-generating procedures such as tracheostomy/nasogastric tube insertion need to be carried out with all due precautions.

\section{Prostate}

Carcinoma of the prostate is relatively slow growing as compared to sites such as head-neck and lung. Zaorsky et $a l^{[20]}$ in their publication have tried to address questions such as whether a subset of patients can have a delay in in-person clinic visit and is there a subset where the treatment can be deferred or avoided and the policies on shortening of treatment. They have proposed the concept of RADS (Remote visit, Avoidance, Deferment, Shortening of RT) for treating prostate cancer patients in this pandemic.

We propose our recommendations based on the guidelines by Zaorsky et al. and the recommendations of the EAU. ${ }^{[21]}$

- Very low-risk group of patients, in the current setting, may avoid RT

- Low-risk and favorable intermediate-risk group of patients needs to delay RT. RT may be of 5/20 Fx

- Unfavorable intermediate-risk group, high-risk, very high-risk group, and node-positive patients need 
$\mathrm{RT}+$ androgen deprivation therapy (ADT). ADT can be used for 4-6 months to delay RT. Six-month depot preparation can be considered. RT may be of 5/20 Fx.

- Adjuvant/salvage treatment: $\mathrm{RT} \pm \mathrm{ADT}$ to be considered and ADT 6month depot can be preferred. RT can be given in 20 Fractions

- Treatment for oligometastatic + low volume metastatic disease: 3-5 Fractions RT \pm ADT 6 month depot can be preferred. Brachytherapy in the current setting has been proposed to be delayed until safe and avoidable in most of the setting currently.

\section{Conclusion}

This publication is aimed at providing an overview of radiation oncology practices to our multidisciplinary team colleagues in the backdrop of the pandemic. We have tried to summarize few sites and subsites with the help of available guidelines and literature. The overall focus of a radiation oncology department should be to prioritize their patients and avoid delays in the treatment in patients who require it the most; further, if simpler plans can achieve similar outcomes, complex intensity-modulated plans could be avoided to optimize resources. Shortening of total treatment duration whenever feasible is of utmost importance as it would avoid resource crunch. Treatment decisions, however, can be modified based on the institutional support staff availability and thorough discussion with both patient and relatives about the possible risk-benefit ratio.

\section{Kaustav Talapatra', Dipanjan Majumder², Sarbani Ghosh Laskar ${ }^{3}$}

${ }^{1}$ Department of Radiation Oncology, Kokilaben Dhirubhai Ambani Hospital, Mumbai, Maharashtra, India, '2Department of Radiation Oncology, Ruby General Hospital, Kolkata, West Bengal, India, ${ }^{3}$ Department of Radiation Oncology, Tata Memorial Hospital, Mumbai, Maharashtra, India

Address for correspondence: Dr. Dipanjan Majumder, Kasba Golpark, E. M. Bypass, Kolkata - 700 107, West Bengal, India. E-mail:dipanjanmajumder47@gmail.com

Submitted: 02-Jun-2020

Revised: 21-Jun-2020

Accepted: 23-Jun-2020

Published: 29-Oct-2020

\section{References}

1. Patil V, Noronha V, Chaturvedi P, Talapatra K, Joshi A, Menon N, et al. COVID-19 and head and neck cancer treatment. Cancer Res Stat Treat 2020;3:15-28.

2. Yu J, Ouyang W, Chua ML, Xie C. SARS-CoV-2 transmission in patients with cancer at a tertiary care hospital in Wuhan, China. JAMA Onco 2020;6:1108-10.

3. Mohler JL, Antonarakis ES. NCCN guidelines updates: Management of prostate cancer. J Natl Compr Canc Netw 2019;17:583-6.

4. Available from: https://www.nice.org.uk/guidance/ng162/ resources/covid19-rapid-guideline-delivery-of- radiotherapypdf-66141897390277. [Last accessed on 2020 Apr 16].

5. Clinical Guide for the Management of Cancer Patients during the Coronavirus Pandemic. NHS. Approval ref no. 001559; $17^{\text {th }}$ March, 2020.
6. Tchelebi LT, Haustermans K, Scorsetti M, Hosni A, Huguet F, Hawkins MA, et al. Recommendations for the use of radiation therapy in managing patients with gastrointestinal malignancies in the era of COVID-19. Radiother Oncol 2020;148:194-200.

7. Gay HA, Santiago R, Gil B, Remedios C, Montes PJ, López-Araujo J, et al. Lessons learned from Hurricane Maria in Puerto Rico: Practical measures to mitigate the impact of a catastrophic natural disaster on radiation oncology patients. Pract Radiat Oncol 2019;9:305-21.

8. Kunkler IH, Williams LJ, Jack WJ, Cameron DA, Dixon JM; PRIME II investigators. Breast-conserving surgery with or without irradiation in women aged 65 years or older with early breast cancer (PRIME II): A randomised controlled trial. Lancet Oncol 2015;16:266-73.

9. Bartelink H, Maingon P, Poortmans P, Weltens C, Fourquet A, Jager $\mathrm{J}$, et al. Whole-breast irradiation with or without a boost for patients treated with breast-conserving surgery for early breast cancer: 20-year follow-up of a randomised phase 3 trial. Lancet Oncol 2015;16:47-56.

10. Haviland JS, Owen JR, Dewar JA, Agrawal RK, Barrett J, Barrett-Lee PJ, et al. The UK Standardisation of Breast Radiotherapy (START) trials of radiotherapy hypofractionation for treatment of early breast cancer: 10-year follow-up results of two randomised controlled trials. Lancet Oncol 2013;14:1086-94.

11. Brunt AM, Haviland J, Sydenham M, Algurafi H, Alhasso A, Bliss $\mathrm{P}$, et al. FAST Phase III RCT of radiotherapy hypofractionation for treatment of early breast cancer: 10Year Results (CRUKE/04/015). Int J Radiat Oncol Biol Phys 2018;102:1603-4.

12. Brunt AM, Wheatley D, Yarnold J, Somaiah N, Kelly S, Harnett A, et al. Acute skin toxicity associated with a 1-week schedule of whole breast radiotherapy compared with a standard 3-week regimen delivered in the UK FAST-Forward Trial. Radiother Oncol 2016;120:114-8.

13. Bernier J, Cooper JS, Pajak TF, van Glabbeke M, Bourhis J, Forastiere A, et al. Defining risk levels in locally advanced head and neck cancers: A comparative analysis of concurrent postoperative radiation plus chemotherapy trials of the EORTC (\#22931) and RTOG (\# 9501). Head Neck 2005;27:843-50.

14. Chandarana MN, Pai PS. Demography and treatment pattern of patients with head and neck carcinoma presenting to a tertiary care center in India: Need for urgent decentralization of cancer care. South Asian J Cancer 2020;9:38-42.

15. Licitra L, Grandi C, Guzzo M, Mariani L, Lo Vullo S, Valvo F, et al. Primary chemotherapy in resectable oral cavity squamous cell cancer: A randomized controlled trial. J Clin Oncol 2003;21:327-33.

16. Nichols AC, Theurer J, Prisman E, Read N, Berthelet E, Tran E, et al. Radiotherapy versus transoral robotic surgery and neck dissection for oropharyngeal squamous cell carcinoma (ORATOR): An open-label, phase 2, randomised trial. Lancet Oncol 2019;20:1349-59.

17. Ghi MG, Paccagnella $\mathrm{A}$, Ferrari $\mathrm{D}$, Foa $\mathrm{P}$, Alterio $\mathrm{D}$, Codecà $\mathrm{C}$, et al. Induction TPF followed by concomitant treatment versus concomitant treatment alone in locally advanced head and neck cancer. A phase II-III trial. Ann Oncol 2017;28:2206-12.

18. Patil VM, Noronha V, Joshi A, Muddu VK, Dhumal S, Arya S, et al. Weekly chemotherapy as induction chemotherapy in locally advanced head and neck cancer for patients ineligible for 3 weekly maximum tolerable dose chemotherapy. Indian $\mathrm{J}$ Cancer 2014;51:20-4.

19. Zhang L, Huang Y, Hong S, Yang Y, Yu G, Jia J, et al. Gemcitabine plus cisplatin versus fluorouracil plus cisplatin in 
recurrent or metastatic nasopharyngeal carcinoma: A multicentre, randomised, open-label, phase 3 trial. Lancet 2016;388:1883-92.

20. Zaorsky NG, Yu JB, McBride SM, Dess RT, Jackson WC, Mahal BA, et al. Prostate cancer radiotherapy recommendations in response to COVID-19. Adv Radia Oncol 2020; [doi: https:// doi.org/10.1016/j.adro. 2020.03.010].

21. Available from: https://uroweb.org/covid-19-recommendationsby-eau-guidelines-panels/. [Last accessed on 2020 Jun 04].
This is an open access journal, and articles are distributed under the terms of the Creative Commons Attribution-NonCommercial-ShareAlike 4.0 License, which allows others to remix, tweak, and build upon the work non-commercially, as long as appropriate credit is given and the new creations are licensed under the identical terms.

\begin{tabular}{|l|l|}
\hline \multicolumn{2}{|c|}{ Access this article online } \\
\hline Quick Response Code: & Website: \\
& www.ijmpo.org \\
\cline { 2 - 2 } & DOI: \\
\hline
\end{tabular}

How to cite this article: Talapatra K, Majumder D, Laskar SG. COVID-19: An advisory for a radiation oncology department pertinent to the multidisciplinary team. Indian J Med Paediatr Oncol 2020;41:629-33. 